\title{
An overview of digital speech watermarking
}

\begin{abstract}
Digital speech watermarking is a robust way to hide and thus secure data like audio and video from any intentional or unintentional manipulation through transmission. In terms of some signal characteristics including bandwidth, voice/non-voice and production model, digital speech signal is different from audio, music and other signals. Although, various review articles on image, audio and video watermarking are available, there are still few review papers on digital speech watermarking. Therefore this article presents an overview of digital speech watermarking including issues of robustness, capacity and imperceptibility. Other issues discussed are types of digital speech watermarking, application, models and masking methods. This article further highlights the related challenges in the real world, research opportunities and future works in this area, yet to be explored fully.
\end{abstract}

Keyword: Digital speech watermarking; Digital speech data hiding; Digital audio and speech watermarking 